\title{
Modelo de gestión presupuestal para mejorar la defensa jurídica en el Gobierno Regional de San Martín
}

\author{
Abraham Mafaldo Macedo \\ amafaldo_19@hotmail.com \\ Universidad César Vallejo
}

\section{RESUMEN}

El presente estudio tiene como propósito la propuesta de un modelo de gestión presupuestal para mejorar la defensa jurídica del Gobierno Regional de San Martín el diagnóstico de la defensa jurídica, el estudio fue de tipo aplicada, por cuanto tuvo como propósito resolver problemas prácticos (CONCYTEC, 2018), tuvo un diseño descriptivo - propositivo (Sampieri, 2014). Además, se observa alcance temporal, transversal y orientada a la aplicación, ya que se centró en obtener datos sobre lo que sucede en un momento único (Gallardo, 2017). Para el estudio se consideró a 23 profesionales abogados que laboran dentro del área de Defensa Jurídica del Gobierno Regional de San Martín. Asimismo, se consideró al jefe del área de Defensa Jurídica, siendo en total 24 colaboradores. Se utilizó como técnica la encuesta, el estudio mostró 2 fases diagnostica y fase propositiva, en ese sentido los resultados enmarcan a la deficiencia en la programación de mayoría de las actividades ejecutadas, lo cual dificulta hacer frente a los requerimientos de los colaboradores y también de la población, evitando de ese modo garantizar el cumplimiento de los objetivos institucionales. Por otro lado, la deficiencia en la programación de los ingresos que se van a obtener y gastos que se van a incurrir durante un periodo de tiempo determinado, sin prestan mucha atención al análisis de los límites máximos a gastarse durante ese periodo, finalmente un modelo contribuye de manera significativa en el desarrollo organizacional.

Palabras clave: gestión, modelo, defensa jurídica, programa de ingreso, presupuesto. 


\title{
Budget management model to improve legal defense in the Regional Government of San Martín
}

\begin{abstract}
:
The purpose of this study is to propose a budget management model to improve the legal defense of the Regional Government of San Martín, the diagnosis of legal defense, the study was of an applied type, since it had the purpose of solving practical problems (CONCYTEC, 2018), had a descriptive - purposeful design (Sampieri, 2014). In addition, a temporal, transversal and application-oriented scope is observed, since it focused on obtaining data about what happens in a single moment (Gallardo, 2017). For the study, 23 professional lawyers who work within the Legal Defense area of the San Martín Regional Government were considered. Likewise, the head of the Legal Defense area was considered, with a total of 24 collaborators. The survey was used as a technique, the study showed 2 diagnostic phases and a propositional phase, in that sense the results frame the deficiency in the programming of most of the activities carried out, which makes it difficult to meet the requirements of the collaborators and also the population, thus avoiding guaranteeing the fulfillment of institutional objectives. On the other hand, the deficiency in the programming of the income that will be obtained and expenses that will be incurred during a certain period of time, without paying much attention to the analysis of the maximum limits to be spent during that period, finally a model contributes significantly to organizational development.
\end{abstract}

Keywords: management, model, legal defense, admission program, budget.

Artículo recibido: 14 oct. 2020

Aceptado para publicación: 04 nov. 2020 Correspondencia:amafaldo_19@hotmail.com, Conflictos de Interés: Ninguna que declarar 


\section{INTRODUCCIÓN}

Es conveniente señalar que la defensa jurídica del Estado comprende una serie de procesos, normas, mecanismos e instrumentos integrados de modo funcional con el objetivo de velar por los intereses del Gobierno ante demandas que puedan desarrollar particulares y demás instituciones frente a procesos planteados en su contra, tal como lo indica Juárez (2016) "su accionar por lo general no es tomado en cuenta al momento de la planificación institucional y se plasma en la poca asignación presupuestal para la ejecución de sus actividades” (p.8). En el contexto internacional, las funciones efectuadas en pro del cumplimiento de los derechos de los gobiernos en su orden interno se encuentra regulado incluso como parte del marco constitucional, así Cantuarias (2014) indica que en el caso chileno la Constitución de 2010 la incluye como un instituto dentro del Ministerio de Justicia, el mismo que presenta autonomía administrativa y presupuestal; por otra parte, en Colombia, la Constitución de 1991, le brinda rango constitucional autónomo, cuyos miembros son elegidos por el Tribunal Constitucional por un periodo de 10 años y renovables.

Basándonos en la conceptualización de justicia, puede señalarse que es un principio concedido a la ciudadanía con el fin de asegurar la convivencia pacífica y considerándolo como un medio indispensable dentro del sistema de derecho democrático; así pues, los procedimientos contenciosos administrativos producen un ambiente de conflicto para la población puesto que el $93 \%$ indican, que estos procesos son tediosos y de larga duración (Grafe, 2016); por lo que se considera necesario determinar procedimientos que faciliten la atención de estos casos. Asimismo, Grafe (2016), alude que la defensa jurídica es considerada como un aspecto relevante dentro de los países de Latinoamérica, tales como Argentina, Chile, Paraguay, México, Brasil, etc., quienes en la mayoría de las circunstancias donde se presentan demandas contra un organismo público o Gobierno en sí, el demandante busca recibir algún tipo de compensación económica como por ejemplo una indemnización o pago por reparación civil que subsane la falta cometida por el Estado en su contra. Es por eso que las falencias percibidas concernientes a la eficiencia y eficacia de las actividades de las agencias de defensa jurídica afectan de manera significativa los recursos públicos de un Gobierno.

Por otro lado, Carrillo \& Varela (2016) aseguran que, a inicios del año 2011, el gobierno de Colombia no contaba con lineamientos y políticas bien definidas que contribuyan con la efectividad de la defensa jurídica y la mejora del funcionamiento de los organismos 
estatales afectados. Frente a la ausencia de tales medidas estratégicas efectivas que prevengan los daños antijurídicos, los litigios en contras de los organismos públicos fueron incrementando de manera significativa, siendo los principales factores determinantes de este crecimiento: La ausencia de una conducta de buen gobierno que contribuya con la solución de los reclamos por parte de la ciudadanía dentro de las instituciones del Estado, la ausencia de herramientas de defensa jurídica que estén articuladas de manera institucional y la falta de medidas estratégicas congruentes, falencias en los procesos de obtención de información para conocer los criterios que se deben tener en cuenta al momento de proceder con la demanda en contra de un organismo público, falencias que se presentan en las pericias efectuadas por los abogados de los organismos públicos, presencia de sectores vulnerables, ausencia de un fondo ante las contingencias que se presentan en relación a conciliaciones, compensaciones, etc., finalmente, la falta de difusión de las normativas jurisprudenciales, suscitando la aparición de situaciones que producen confusión del marco legislativo.

En el caso peruano, se reconoce que el Sistema de Defensa Jurídica, el mismo que se encuentra regulada por D.L. $\mathrm{N}^{\circ} 1068$ y D.S. $\mathrm{N}^{\circ} 017-2008-J U S$, por medio del cual se establece la forma como el Estado debe llevar a cabo su defensa ante hechos que le atañen; sin embargo, este accionar tiene limitaciones. Juárez (2016) indica que existe una alta carga procesal sin resolver, poca disponibilidad presupuestal, alta probabilidad de obtener sentencias desfavorables al Estado, incumplimientos de las sentencias, que la postre acarrea desazón y malestar de los usuarios.

La problemática expuesta y analizada desde el enfoque de la gestión presupuestal, (MINJUS, 2017) indica que las sentencias judiciales producto de demandas de los administrados en el Poder Ejecutivo representa 7,643 millones de soles, siendo los sectores educación y salud quienes tiene a deuda mayor, por consiguiente esta situación al ser muy elevada no pueda ser cumplida a cabalidad, ocasionando a su vez distorsiones presupuestales, afectando el cumplimiento de las metas operativas de las instancias gubernamentales y reclamos de los administrados para su cumplimiento.

Esta situación viene siendo abordada desde el contenido del Decreto Supremo 004-2013PCM, en donde se diseñan políticas nacionales en pro de la modernización de la gestión pública, por cuanto el sector justicia reconoce que la defensa jurídica debe responder a criterios de carga procesal y alineados a los programas presupuestales del Estado. Sin 
embargo, en correspondencia con la Ley $\mathrm{N}^{\circ} 27867$ se busca defender judicialmente los intereses del Gobierno y quienes actúen en nombre de él, y desarrolla sus funciones con los entes rectores nacionales sólo relaciones de coordinación y cooperación, lo que limita su accionar, sobre todo cuando muchas de los procesos tienen carácter nacional en cuanto a las demandas que se plantean.

De acuerdo a la planificación del gasto, se determina el plazo para su ejecución, los recursos que se van emplear, lineamientos a seguir, objetivos y acciones de contingencia que se deben tener en cuenta, los cuales deben ser presentados en base al Presupuesto Multianual asignado a la institución para un año fiscal establecido. Por otro lado, el decreto en mención señala que se es conveniente identificar y estudiar las cualidades y rasgos internos de un organismo, como también se busca analizar y plantear acciones correctivas asertivas frente a las mismas. Sin embargo, se percibe que no se está considerando lo mencionado con anterioridad, por cuanto no se identifica de manera oportuna las debilidades del gobierno regional, y por ende no se están tomando medidas para contrarrestar las deficiencias. Como la falta de planificación de gastos que afectan directamente a los colaboradores, de los cuales sus reclamaciones no se resuelven en el tiempo conveniente, la ausencia de fondos de contingencia suficientes para hacer frente a los depósitos por concepto de conciliación, laudo o sentencia, además de otras falencias concernientes al conocimiento empírico de la defensa jurídica de los profesionales en abogacía producido por la falta de capacitaciones constantes.

Desde un contexto regional, se evidencia que al 2017 la deuda social como producto de sentencias firmes producto de litigios asumidos por la defensa jurídica del Gobierno Regional de San Martín ascendió a 865.13 millones, representando un 113\% del presupuesto regional para el periodo 2016 (MEF, 2018), lo cual hace que, desde el enfoque de la gestión presupuestal, sea imposible su cumplimiento en el corto plazo, pues ello, a pesar de su cumplimiento parcial, genera distorsiones de orden presupuestal o mayores asignaciones de créditos suplementarios, acciones que no son entendidas por los administrados, y que escapan a las competencias y atribuciones que tiene el gobierno regional para su cumplimiento.

Así para el año 2018, el Gobierno Regional de San Martín (GORESAM) obtuvo un crédito presupuestario suplementario por un importe ascendente a 78.16 millones para cumplir con la deuda social, (MEF, 2018), principalmente para el sector salud y educación; sin embargo 
el tema de cumplimiento se agrava debido a que año tras año se dan nuevas sentencias y este monto de la deuda se incrementa y no disminuye; montos a los cuales se tienen que adicionar las deudas que tiene el Gobierno Regional producto de arbitrajes y conciliaciones por los procedimientos establecidos en el Marco de Ley de Contrataciones, muchas de las cuales tienen nivel de obligación forzosa mediante embargos judiciales, lo que genera problemas de cumplimientos de metas presupuestales y de gestión institucional acorde con lo planificado.

La gestión presupuestal del GORESAM enfocada a la gestión de la defensa jurídica es escasa, asignando según su portal electrónico, un presupuesto para el año 2017 de s/.318,014 para un total de 10 abogados defensores y 06 administrativos, aun cuando la carga procesal supera todas las ratios de eficiencia y productividad, lo que genera que los casos no sean asumidos con calidad y en el $96.4 \%$ las sentencias son desfavorables para el mismo (Mafaldo, 2017).

Desde esta perspectiva, el presente estudio pretende proponer estrategias de gestión presupuestal para mejorar la defensa jurídica del Estado en el Gobierno Regional de San Martín, a través del análisis de las dimensiones e indicadores para determinar recomendaciones para su mejora, como parte de una gestión pública basada en la eficiencia y calidad. De ello nació como formulación del problema, ¿Qué modelo de gestión presupuestal se debe implementar para mejorar la defensa jurídica del Gobierno Regional de San Martín, año 2020?, en ese sentido se consideró conveniente plantear como objetivo general: Proponer un modelo de gestión presupuestal para mejorar la defensa jurídica del Gobierno Regional de San Martín.

Un análisis de información, es el abordaje de contexto internacional presentado por Gutiérrez (2016) quien afirma que se reconoce que la defensa jurídica está estrechamente relacionada con el nivel de responsabilidad que debe asumir el gobierno frente a las situaciones que se presentan de carácter jurídico, debido a que ambos están enfocados a resarcir daños y direccionar las acciones del gobierno hacia el bien común. Así pues, al articular la defensa del Estado se busca algo más que evadir o eludir responsabilidades a cargo de sus fines o funciones, en atención de la propia ejecución de los casos o sentencias, puesto que no se tiene en cuenta los principios de equilibrio y estabilidad que debe aplicar el Estado, y que implica para las cortes, una revisión tanto del derecho y la economía. En tanto, Urrego (2016) concluye que, de acuerdo con el organismo competente responsable 
de la defensa jurídica del Gobierno Colombiano, los procesos para prevenir la presencia de daños jurídicos consisten en identificar un inconveniente, diseñar acciones correctivas para subsanar los inconvenientes, costear las acciones correctivas y elaborar planes para garantizar su correcta realización y monitoreo constante. Es preciso señalar además que los daños antijurídicos afectan tanto a las finanzas del Estado como a la credibilidad de los funcionarios responsables de ejecutar estas acciones. Por otra parte, se detectó incidencia entre ambos temas de estudio, por lo cual se debe tener en cuenta establecer estrategias gubernamentales y acciones administrativas eficaces mediante un poder ético.

De la misma manera, Martin (2016) indica que en el caso estudiado se ha podido evidenciar que una las razones por las cuales se puede declarar nulo el procedimiento penal es por la falta de eficiencia de la defensa jurídica conforme a lo señalado en la Ley $\mathrm{N}^{\circ} 906$. Al respecto, las agencias no tienen la probabilidad de disponer un abogado durante el desarrollo del procedimiento penal y además en aquellas circunstancias en las cuales en las que su presencia no sea eficiente, es decir, cuando no percibe que no está capacitada en debida forma y tampoco cuenta con el conocimiento adecuado y suficiente para debatir lo expuesto por la parte acusadora. Parra (2017) indica que el diseño de políticas públicas en pro de la defensa jurídica no es resultado de una fuerza política o social, si no se origina como producto del incremento significativo de hechos jurídicos, en donde los actores principales fueron las instituciones estatales y órganos internacionales; por lo que la implantación de estas políticas estuvo enfocada generalmente a las instituciones nacionales. Referente a ello, la implantación de estas políticas no ha representado cambios significativos dentro del modelo de gestión para el periodo 2002-2014 puesto que para defender los intereses de las instituciones estatales se contrataron abogados que presentan un perfil profesional básico.

Por otro lado, a nivel nacional Acuña \& Ángeles (2017) indican que el SDJE no se encuentra debidamente configurado en función a los aspectos organizacionales y funcionales que implican la eficacia y eficiencia de la procuraduría para promover su efectividad. En ese contexto, se reconoce que la implementación del sistema de defensa es deficiente debido a la poca disponibilidad presupuestal y funcional lo cual suscita un aumento de juicios en contra de las instituciones y entidades estatales; asimismo, no existe una mejora de la escala remunerativa para los profesionales cuyo propósito principal es asegurar el dictamen de sentencias favorables para el Gobierno con el fin de atraer a 
individuos debidamente capacitados y competentes. Piña (2017) concluye que la gestión de la Municipalidad de Maynas es deficiente a causa de las numerosas cargas procesales existentes, reducido número de profesionales calificados y competentes para plantear soluciones y la poca asignación de presupuesto asignado para ello. El valor remunerativo de los profesionales se encuentra muy por debajo de lo percibido en el mercado laboral, lo cual reduce las opciones para contar con personas capacitadas y con la experiencia suficiente, pues el $82 \%$ de las cargas procesales son originadas por los colaboradores en cuanto al pago respectivo de sus beneficios. Por lo que se reconoce la necesidad de implantar un modelo de gestión que contribuya con la optimización del funcionamiento de la entidad. Sin embargo, Villar (2016) concluye que reconoce que la gran parte de abogados no cuenta con el conocimiento empírico necesario para ser partícipe de los procedimientos penales, demostrando así la falta de capacitación de los mismos, por lo que se deduce que el ejercicio de defensa jurídica es deficiente. Por ello, se percibe la necesidad de promover institucionalmente este derecho destacando su importancia. Saavedra (2017) concluye que la relación entre la defensa jurídica y la corrupción es directa y significativa con un nivel de correlación de 0.564 , pues el grado de eficiencia de la defensa jurídica es de $18.1 \%$ y de la valoración de la corrupción de servidores y funcionarios públicos 2016 es deficiente donde el $21.9 \%$ indica que esto ocurre siempre y casi siempre. La defensa jurídica se encuentra inmersa en distintos procesos, lo cual imposibilita el logro de las metas institucionales.

El estudio se fundamenta en teorías y enfoques conceptuales relacionados con las variables en estudio; referente a la evolución histórica de la Gestión de la Defensa Jurídica, Guzmán (2016) refiere que la historia se remonta al derecho francés donde durante el siglo XVIII ante el surgimiento de los derechos colectivos y siendo el Estado un garante de ellos y en muchos casos el que los vulnera, se hacía necesario contar con un ente encargado de velar la aplicación de tales derechos. Por su parte Juárez (2016) indica que, en el caso peruano, las Constitución Política del siglo XIX y XX, al referirse a la defensa jurídica se menciona a los "agentes fiscales", los mismos que eran los responsables de defender los intereses públicos, en ese sentido presenta información fehaciente que permite abordar los conceptos básicos e histórico de los procedimientos en estudio. Asimismo, Cruz (2019), menciona que es un tipo de protección que se basa en defender a un individuo o institución frente a un problema jurídico o reclamaciones. Guzmán (2016) indica que la Constitución de 1978 
no incorpora la defensa jurídica del Estado, y por consiguiente se mantiene con un rango de Ley. Además, en el Art.47 de la Carta Magna Peruana de 1993 se encuentra tipificado que las personas encargadas de defender los intereses públicos son los Procuradores Públicos, los mismos que dependen del sector justicia.

Además, en el Art.46 de la Ley N²9158 está determinado que los sistemas administrativos tienen como objetivo principal asegurar la utilización efectiva de los recursos estatales y, específicamente en el inc.10 detalla que uno de estos sistemas corresponde a la Defensa Judicial del Estado (Juárez, 2016). Para el año 2008, la creación del Sistema de Defensa Jurídica mediante el D.S. N017-2008-JUS, en donde según lo tipificado en el Art.2 se deduce que este sistema está comprendido por lineamientos, principios, normativas, procesos, etc., los mismos que facilitarán el desarrollo de las funciones de los procuradores mediante el ejercicio de defensa jurídica (Guzmán, 2016).

En concordancia con la premisa anterior, la gestión de la defensa jurídica "comprende una serie de procedimientos o tendencias administrativas y legales sustentadas en el marco normativo, documentos de gestión y mecanismos integrados mediante actividades o programas concretos”. (MINJUS, 2017). Así pues, este procedimiento como tal representa los procedimientos claves para que el profesional pueda desarrollar apropiadamente frente a cada caso que se presente. Del mismo modo es aquella actividad ejercida por los procuradores y el Sistema Jurídico en general que comprende al Presidente del Consejo, miembros del consejo de defensa jurídica, procuradores y presidente del tribunal de sanción (MINJUS, 2017). Pachot (2016) menciona que se constituye de todas las actuaciones que la ley en termino procesal y arbitral permiten. Seguidamente, Vásconez (2017), menciona que constituye normas, procesos administrativos. Crea e integra las leyes, estándares, tecnologías e instrumentos a través de métodos y procedimientos específicos.

Arieta (2017) sostiene que la creación del Sistema de Defensa Jurídica del Estado - SDJE fue efectuado a través del Decreto Legislativo 1068 para defender los intereses públicos y garantizar que las actividades ejecutadas estén unificadas en todos sus niveles y todas sus particularidades. Por otro lado, en el mes de enero del año 2017, dicho decreto fue derogado por medio de D.L. $\mathrm{N}^{\circ} 1326$, mediante el cual se realiza la reestructuración del Sistema Administrativo de Defensa Jurídica y la creación de la Procuraduría General del Estado, asimismo fue aprobado su reglamento a través del D.S 018-2019-JUS en el mes de noviembre del año 2019, la misma que ejerce autonomía en lo que respecta a sus aspectos 
funcionales, técnicos, económicos y administrativos con el propósito de garantizar su correcto funcionamiento. Con respecto a los factores que afectan la defensa jurídica, Aguado \& López (2016), indican que están agrupados desde dos enfoques, el primero administrativo y el segundo funcional. Por otro lado, los Modelos de gestión actual sobre la Defensa Jurídica en función de la organización del sistema de defensa jurídica se tienen los siguientes modelos de gestión: a) Modelo Centralizado: Está caracterizado por concentrar la función de defensa jurídica sobre un conjunto de profesionales de derecho pertenecientes al sector público con la finalidad de que la agencia sea un órgano responsable de planificar e implementar mecanismos para la defensa del Estado (De Oliveira, 2016). Por ello, la prestación de servicios jurídicos no corresponde a las facultades respectivas, las mismas que son caracterizadas por ser autónomas de tal manera que se puedan hacer frente a una demanda (Kaneberg \& Jensen, 2019)., b) Modelo Mixto: Está caracterizado por la existencia de un grupo de profesionales de derecho pertenecientes al sector público, con la diferencia de que algunos de estos son descentralizados en las acciones según el ciclo de defensa, reflejando la jerarquía existente (Veleño, 2016). Por otro lado, busca establecer una similitud con el sistema del Estado, enfocado en un sistema unitario considerado como el centro de todas las funciones determinadas desde el mismo centro (De Pontes \& Corneli, 2017). Por último, el Modelo Descentralizado: Está caracterizado por contar con una agencia en donde los profesionales desarrollan labores más concentradas en comparación con los dos modelos anteriores, en donde la institucionalidad se constituye por una gran cantidad de participantes sin que puedan afectar de algún modo a los mismos por las labores que realizan (Herz, 2018).

Haciendo referencia a la gestión presupuestal, se menciona que la estrategia de gestión presupuestal se realiza a través de las herramientas e instrumentos de gestión establecidos en las leyes de presupuesto y supletoriamente de aplicación las leyes del derecho administrativo, de contrataciones, control interno, contaduría pública, entre otras, entre las cuales deben ir orientadas a la defensa jurídica (Cano, 2018). El Presupuesto Público, es un instrumento que permite la distribución eficiente de los recursos del Estado y permite evaluar que se alcancen los resultados provechosos para la sociedad, por ello es importante definir bien las metas y objetivos institucionales y la responsabilidad que se deberá asumir para alcanzarlos (Gutiérrez \& Peñaloza, 2017). Su cumplimiento se evalúa en función al avance físico, su elaboración garantiza que las instituciones u organismos cumplan con los 
proyectos de inversión y mejora la calidad de vida poblacional. Por otra parte, el presupuesto público no sólo asigna recursos, sino que determina las personas competentes para su ejecución de las acciones, los procesos que se deberán seguir y los medios que se emplearán para alcanzar los objetivos, así también ayuda con la rendición de cuentas, siendo detallado en la Ley del Presupuesto Público, Plan Operativo Anual y Presupuesto Institucional, parte de este presupuesto debe ser destinado en diferentes actividades de la defensa jurídica como fuente de apoyo (Aguilar \& Díaz, 2020).

Con respecto a la importancia de la gestión presupuestal, Bernal (2016), manifiesta que, la gestión eficaz del presupuesto no solo ayuda a gestionar mejor el efectivo de una institución, sino que también ayuda a: Controlar sus resultados financieros para que pueda hacer ajustes si es necesario. Asimismo, Cauvin (2018), alude que la gestión presupuestal es importante porque alinea el impacto financiero con otros factores de importancia en las operaciones para evitar errores. Por último, Klein y Lemos (2019), manifiesta que es de relevancia porque promueve la evaluación del desempeño, la cual supone la única forma de maximizar el valor del dinero y los activos disponibles.

El Presupuesto Público y estrategias para su implementación, la finalidad del presupuesto es garantizar que su ejecución genere un impacto positivo en la sociedad, ya sea proporcionando bienes o servicios de calidad, generando una calidad de gasto eficiente y mejorando el desempeño del Gobierno (Arana \& Ramírez, 2019). Para ello, es esencial que se planifique estratégicamente el funcionamiento del Estado en sus distintos niveles, y su correlato con los presupuestos institucionales constituyen las herramientas básicas para la implementar el presupuesto público adicionando la intervención de la ciudadanía a través del presupuesto participativo (Arrais, 2019).

Con respecto a las tendencias de los modelos de gestión, las transformaciones en la acción del Estado aparecieron diversas corrientes teóricas, doctrinales e ideológicas que abordaron el análisis de la burocracia y avalaron, justificaron o criticaron los cambios sufridos en el aparato burocrático público y en las orientaciones del Estado. Frente a ello, el Gobierno tuvo que asumir nuevos roles y ampliar aquellos que ya se encontraban bajo su control y supervisión. A partir del siglo XX, las entidades estatales se ocupaban principalmente de ejecutar obras públicas en bien de la población y de diseñar proyectos de inversión que mejoren el sector de educación y salud. Desde la perspectiva de la red pública, está la idea 
de que todo bien público debe satisfacer los requerimientos de la población ha ocasionado que estos sean focalizados como perceptores (Stella, 2017).

La gestión presupuestal por medio de sus diversos programas busca la mejora de la defensa jurídica, donde este destina fondos monetarios para ello, contribuyendo con la creación de una defensa efectiva que vigile los intereses públicos por medio de acciones que permitan dirigir e integrar la defensa jurídica, la reparación civil, la representación en los procesos de inconstitucionalidad, competencial y acción popular; así como frente a los organismos supranacionales que buscan proteger el ejercicio de los derechos humanos (Pantarollo, 2019). En consecuencia, este podría caer en déficit, donde se puede destacar déficit primario, el cual es el déficit total menos pagos por intereses, déficit estructural; es el que se deriva de las modificaciones en el nivel de gastos públicos, déficit cíclico; el que se produce como consecuencia de una recesión de la economía, aunque el nivel de gasto público y de ingreso público permitiesen alcanzar el equilibrio presupuestario (Roitbarg, 2018).

Por ello, el modelo de gestión debe contener medidas estratégicas y preventivas frente al daño antijurídico, las mismas que deben ser diseñadas en función a los procesos administrativos y capacidades de los servidores estatales, instalación de sistemas de información que faciliten el desarrollo de una gestión eficiente de los procedimientos y juicio, y mejor capacidad para comprender las medidas de defensa frente a procesos jurídicos por parte de los profesionales de la abogacía (Carvalho \& Mazzon, 2019). Asimismo, el modelo debe presentar un alto nivel de calidad en los trabajos efectuados y la capacidad de respuesta necesaria para el cumplimiento de los estándares de calidad establecidos y el periodo de tiempo requerido. Generalmente, se reconoce la existencia de tres modelos organizativos, los cuales son centralizado, mixto y centralizado; quienes parten de un enfoque funcional y de mando hasta que se representa en un esquema en donde estas se encuentran mejor distribuidas (Gómez, 2017).

\section{ESTRATEGIAS METODOLÓGICAS O MATERIALES Y MÉTODOS}

El estudio fue de tipo aplicada, por cuanto tuvo como propósito resolver problemas prácticos (CONCYTEC, 2018), tuvo un diseño descriptivo - propositivo (Sampieri, 2014). Además, se observa alcance temporal, transversal y orientada a la aplicación, ya que se centró en obtener datos sobre lo que sucede en un momento único (Gallardo, 2017). 
Gallardo (2017), la investigación ejecutada fue propositiva, además es no experimental, porque para su desarrollo no se manipulo las variables en estudio y fueron estudiadas, analizadas y evaluadas tal y como se presentan en el ámbito de investigación (Monje, 2015). En cuanto a los participantes, Valderrama (2016) indica que la población es el conjunto de individuos o grupo poblacional que se encuentra en un espacio geográfico medible y cuantificable, en ese sentido para el estudio se consideró a 23 profesionales abogados que laboran dentro del área de Defensa Jurídica del Gobierno Regional de San Martín. Asimismo, se consideró al jefe del área de Defensa Jurídica, siendo en total 24 colaboradores. La muestra tal como menciona Valderrama (2016) es la parte representativa de un conjunto, que permite la evaluación mediante el uso de herramientas, de acuerdo a ello, la muestra estuvo conformada por la totalidad de la población, es decir por 23 abogados encargados de la Defensa Jurídica, y al jefe del área de la Defensa Jurídica del Gobierno Regional de San Martín, siendo en total 24 colaboradores, es decir el 100\% de la población.

Se utilizó como técnica la encuesta, la misma que, según lo determinado por Sampieri (2014), es aplicable a una investigación que pretende conocer las características de una población a partir de definir de forma previa las cualidades o atributos que se quieren conocer, en este caso, la gestión presupuestal del Gobierno Regional de San Martín. Otra técnica que se aplico fue el análisis documental, el mismo que según Sampieri (2014), determina que se aplica cuando se quiere identificar los elementos que conforman una variable en documentos o evidencias ya existentes, de forma tal que guíe el recojo de información de forma ordenada y sistemática.

El estudio mostró 2 fases, fase diagnostica: Como primer paso se elaboró el análisis de la problemática actual sobre la defensa jurídica en la gestión presupuestal del Gobierno Regional de San Martín, asimismo, a través de cada instrumento que fue aplicado, se pudo conocer los problemas de la entidad. Seguidamente, con la información que se recopile, se pudo ejecutar la investigación y se respondió de manera efectiva a cada objetivo propuesto, de la misma forma, se utilizó el programa estadístico SPSS 25, empleando un análisis cuantitativo donde fue necesario emplear tablas y figuras. Fase propositiva: Posterior a la fase diagnostica, se elaboró una propuesta de gestión presupuestal con la intención de mejorar la defensa jurídica en el Gobierno Regional de San Martín, con el propósito de 
reducir los problemas que puedan encontrarse, asimismo obtener mejores resultados en el futuro.

\section{RESULTADOS Y DISCUSIÓN}

Caracterizar la gestión de la defensa jurídica del Gobierno Regional de San Martín

Tabla 1.

Caracterización de la Gestión de la defensa jurídica

\begin{tabular}{|c|c|c|c|c|c|c|c|}
\hline \multirow[t]{2}{*}{ Din } & \multirow[t]{2}{*}{ mensiones/Indicadores } & \multicolumn{2}{|c|}{$\begin{array}{c}\text { Muy } \\
\text { deficiente / } \\
\text { Deficiente }\end{array}$} & \multicolumn{2}{|c|}{ Regular } & \multicolumn{2}{|c|}{$\begin{array}{l}\text { Bueno / } \\
\text { Muy } \\
\text { bueno }\end{array}$} \\
\hline & & fi & $\%$ & fi & $\%$ & fi & $\%$ \\
\hline \multirow{4}{*}{ Planificación } & $\begin{array}{l}\text { Planes operativos elaborados con } \\
\text { participación de los trabajadores }\end{array}$ & 15 & $62.5 \%$ & 4 & $16.7 \%$ & 5 & $20.8 \%$ \\
\hline & $\begin{array}{l}\text { Plan Operativo de la Defensa } \\
\text { Jurídica alineada al PE del } \\
\text { GRSM. }\end{array}$ & 11 & $45.8 \%$ & 8 & $33.3 \%$ & 5 & $20.8 \%$ \\
\hline & $\begin{array}{l}\text { Calificación del plan operativo } \\
\text { en función a lineamientos } \\
\text { programáticos del GRSM. }\end{array}$ & 9 & $37.5 \%$ & 6 & $25.0 \%$ & 9 & $37.5 \%$ \\
\hline & $\begin{array}{l}\text { Planes operativos con asignación } \\
\text { presupuestal independiente y } \\
\text { autónoma. }\end{array}$ & 12 & $50.0 \%$ & 11 & $45.8 \%$ & 1 & $4.2 \%$ \\
\hline \multirow{4}{*}{ Organización } & $\begin{array}{l}\text { Consignación de abogados para } \\
\text { la defensa jurídica. }\end{array}$ & 20 & $83.3 \%$ & 3 & $12.5 \%$ & 1 & $4.2 \%$ \\
\hline & $\begin{array}{l}\text { Consignación de personal } \\
\text { auxiliar a cargo. }\end{array}$ & 10 & $41.7 \%$ & 5 & $20.8 \%$ & 9 & $37.5 \%$ \\
\hline & $\begin{array}{l}\text { Consignación del presupuesto } \\
\text { para llevar a cabo la defensa } \\
\text { jurídica. }\end{array}$ & 3 & $12.5 \%$ & 12 & $50.0 \%$ & 9 & $37.5 \%$ \\
\hline & $\begin{array}{l}\text { Consignación de procedimientos } \\
\text { administrativos en la defensa } \\
\text { jurídica. }\end{array}$ & 17 & $70.8 \%$ & 1 & $4.2 \%$ & 6 & $25.0 \%$ \\
\hline
\end{tabular}


$\begin{array}{lllllllll}\text { Establecimiento de casos en } & 15 & 62.5 \% & 2 & 8.3 \% & 7 & 29.2 \%\end{array}$ cumplimiento de sentencia

Establecimiento de casos con apersonamiento $\begin{array}{llllll}8 & 33.3 \% & 5 & 20.8 \% & 11 & 45.8 \%\end{array}$

Dirección Establecimiento de casos que tuvieron allanamiento $\begin{array}{llllll}16 & 66.7 \% & 3 & 12.5 \% & 5 & 20.8 \%\end{array}$ Establecimiento de casos con procesos dilatados $\begin{array}{llllll}15 & 62.5 \% & 2 & 8.3 \% & 7 & 29.2 \%\end{array}$

Determinación de los casos con apelación.

$15 \quad 62.5 \% \quad 4 \quad 16.7 \% \quad 5 \quad 20.8 \%$

$\begin{array}{lllllll}\text { Sistema de monitoreo de casos } & 17 & 70.8 \% & 3 & 12.5 \% & 4 & 16.7 \%\end{array}$ implementado

Sistema de evaluación de

$\begin{array}{llllll}10 & 41.7 \% & 6 & 25.0 \% & 8 & 33.3 \%\end{array}$ sentencias implementado

Quejas y reclamos de los administrados sobre los casos $\begin{array}{llllll}6 & 25.0 \% & 10 & 41.7 \% & 8 & 33.3 \%\end{array}$ Informes de auditoría desarrollados

Fuente: Cuestionario aplicado a los abogados de la Procuraduría del Gobierno Regional San Martín.

De acuerdo a los resultados de la encuesta: Del 100\% (24) de abogados que laboran en la procuraduría del Gobierno Regional de San Martín, el 83,3\% (20) consideran muy deficiente y deficiente la gestión de consignación de abogados para la defensa jurídica, debido a que se encuentran supeditados a un tema presupuestal; al igual que al sistema de monitoreo de casos implementados y a la consignación de procedimientos administrativos en la defensa jurídica en un 70,8\% (17). Asimismo, en un $66.7 \%$ (16), el establecimiento de casos que tuvieron allanamiento es muy deficiente y deficiente. Conjuntamente, son muy deficientes y deficientes en un $62.5 \%$ (15) los planes operativos elaborados con participación de los trabajadores, los establecimientos de casos en cumplimiento de sentencia, establecimientos de casos con procesos dilatados y la determinación de los casos con apelación. 
Sumando a todo esto, los planes operativos con asignación presupuestal independiente y autónoma y los informes de auditoría desarrollados son muy deficientes y deficientes en un $50.0 \%$ (12), debido a que no se puede evaluar nuevas contrataciones de profesionales o asistentes en el área de defensa jurídica, por un tema presupuestal deficiente; paralelamente a ello, la consignación del presupuesto para llevar a cabo la defensa jurídica es regular en un $50.0 \%$ (12) a causa de que no se puede evaluar nuevas contrataciones de profesionales o asistentes en el área, por un tema presupuestal deficiente. Y, por último, el Plan Operativo de la Defensa Jurídica alineada al PE del GRSM es considerado muy deficiente y deficiente por los abogados y funcionario en un $45.8 \%$ (11), debido a que no se definieron totalmente las actividades o acciones operativas, respectivamente el PEI 2021-2025, no se encuentra considerado en sus objetivos estratégicos mejorar la defensa jurídica del Estado.

Ante ello, se puede afirmar que la mayoría de los abogados consideran que la planificación de los planes operativos, estratégicos y actividades no se están manejando de manera correcta, asimismo debido a la poca eficiencia en la determinación de casos en cumplimiento de sentencia, apersonamiento, allanamiento, entre. Además, el plan operativo en función de lineamientos programáticos es deficiente ya que no se consideran en mejorar el tema presupuestal, en consecuencia, no se avizora de ejercer la debida defensa jurídica.

Caracterizar el estado actual de la gestión presupuestal de la defensa jurídica del Gobierno Regional de San Martín

Tabla 2

Caracterización de la Gestión Presupuestal

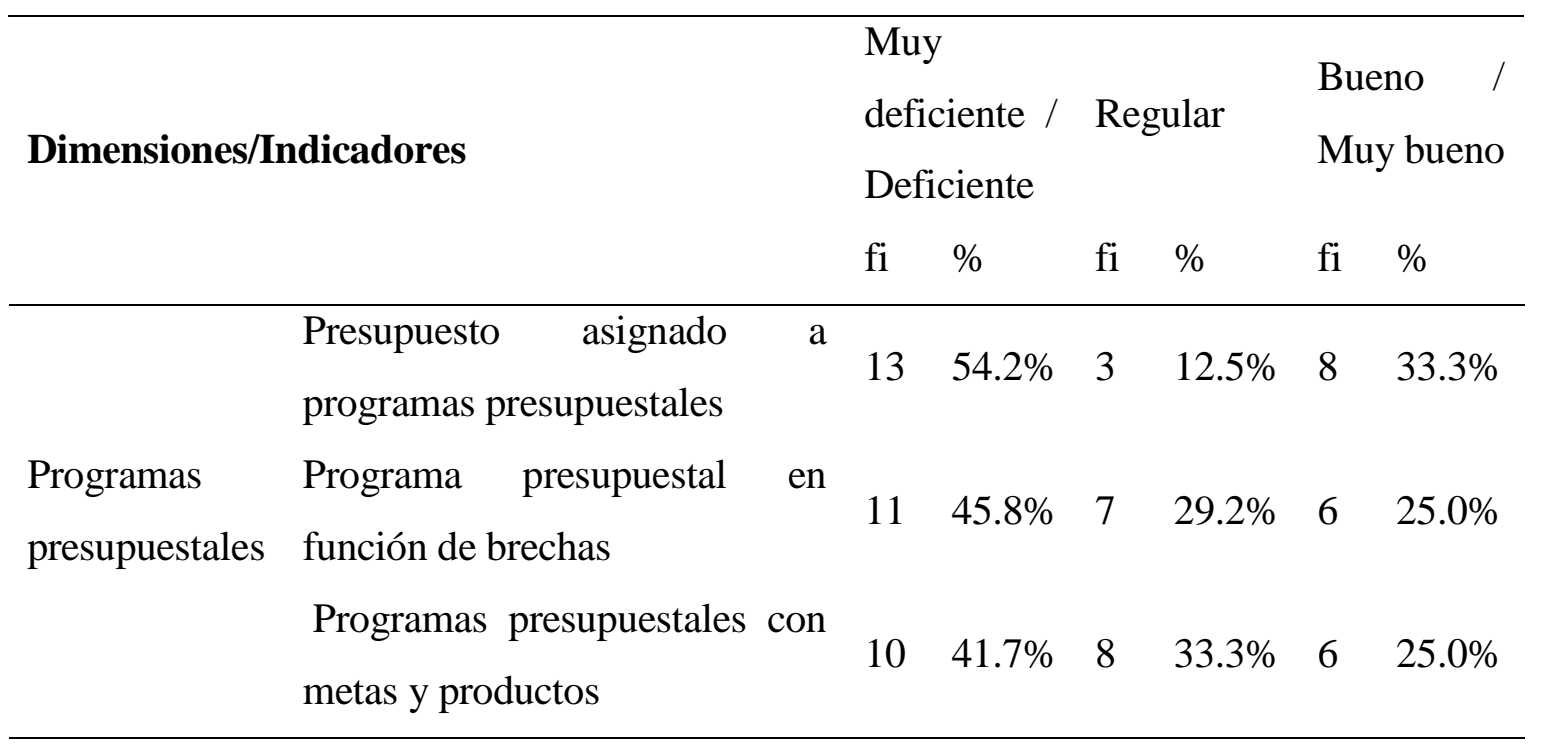




\begin{tabular}{|c|c|c|c|c|c|c|c|}
\hline & $\begin{array}{l}\text { Uso de evidencias y diseños } \\
\text { causales en los programas } \\
\text { presupuestales }\end{array}$ & 14 & $58.3 \%$ & 6 & $25.0 \%$ & 4 & $16.7 \%$ \\
\hline \multirow{4}{*}{$\begin{array}{l}\text { Ejecución } \\
\text { presupuestaria }\end{array}$} & Ingresos en función de POI & 12 & $50.0 \%$ & 10 & $41.7 \%$ & 2 & $8.3 \%$ \\
\hline & Calendario de gasto según POI & 16 & $66.7 \%$ & 5 & $20.8 \%$ & 3 & $12.5 \%$ \\
\hline & $\begin{array}{l}\text { Atención de gastos en función } \\
\text { de PAC }\end{array}$ & 9 & $37.5 \%$ & 6 & $25.0 \%$ & 9 & $37.5 \%$ \\
\hline & Devengado del gasto según POI & 7 & $29.2 \%$ & 6 & $25.0 \%$ & 11 & $45.8 \%$ \\
\hline \multirow{3}{*}{ Seguimiento } & $\begin{array}{l}\text { Monitoreo de indicadores de } \\
\text { Programas presupuestales }\end{array}$ & 14 & $58.3 \%$ & 4 & $16.7 \%$ & 6 & $25.0 \%$ \\
\hline & $\begin{array}{l}\text { Información de indicadores de } \\
\text { programas presupuestales } \\
\text { reportados }\end{array}$ & 14 & $58.3 \%$ & 2 & $8.3 \%$ & 8 & $33.3 \%$ \\
\hline & $\begin{array}{l}\text { Directivos involucrados en el } \\
\text { seguimiento presupuestal }\end{array}$ & 14 & $58.3 \%$ & 2 & $8.3 \%$ & 8 & $33.3 \%$ \\
\hline \multirow{4}{*}{ Evaluación } & $\begin{array}{l}\text { Cumplimiento del propósito de } \\
\text { los programas presupuestales }\end{array}$ & 13 & $54.2 \%$ & 5 & $20.8 \%$ & 6 & $25.0 \%$ \\
\hline & $\begin{array}{l}\text { Evaluación del gasto por } \\
\text { funciones del gasto }\end{array}$ & 8 & $33.3 \%$ & 8 & $33.3 \%$ & 8 & $33.3 \%$ \\
\hline & $\begin{array}{l}\text { Evaluación del gasto por } \\
\text { genéricas del gasto }\end{array}$ & 16 & $66.7 \%$ & 2 & $8.3 \%$ & 6 & $25.0 \%$ \\
\hline & $\begin{array}{ll}\text { Retroalimentación } & \text { de } \\
\text { programas en función de } \\
\text { evaluación }\end{array}$ & 13 & $54.2 \%$ & 4 & $16.7 \%$ & 7 & $29.2 \%$ \\
\hline
\end{tabular}

Fuente: Cuestionario aplicado a los abogados de la Procuraduría del Gobierno Regional San Martín.

Según los resultados obtenidos refieren que: Del 100\% (24) de abogados que laboran en la procuraduría del Gobierno Regional de San Martín, el 66.7\% (20) señalan que la evaluación del gasto por genéricas y el calendario de gasto según POI son deficientes y muy deficientes. De la misma forma, el monitoreo de indicadores de programas presupuestales, 
la información de indicadores reportados, el uso de evidencias y diseños causales en los programas presupuestales y la gestión de los directivos involucrados en el seguimiento presupuestal son muy deficientes y deficientes en un $58.3 \%$ (14). De forma similar son muy deficientes y deficiente en un 54.2\% (13), la retroalimentación de programas en función de evaluación, el cumplimiento del propósito de los programas presupuestales y la gestión del presupuesto asignado a programas presupuestales. Asimismo, la gestión de los ingresos en función de POI es muy deficiente y deficiente 50.0\% (12), respectivamente la gestión del programa presupuestal en función de brechas es muy deficiente y deficiente en un $45.8 \%$ (10) debido a que no se hizo ajuste de personal desde inicio del 2019 a la fecha, y cada profesional abogado se adecua a las funciones encomendadas. Como también los programas presupuestales con metas y productos en un $41.7 \%$ (10).

Los resultados enmarcan a la deficiencia en la programación de mayoría de las actividades ejecutadas, lo cual dificulta hacer frente a los requerimientos de los colaboradores y también de la población, evitando de ese modo garantizar el cumplimiento de los objetivos institucionales. Por otro lado, la deficiencia en la programación de los ingresos que se van a obtener y gastos que se van a incurrir durante un periodo de tiempo determinado, sin prestan mucha atención al análisis de los límites máximos a gastarse durante ese periodo. Otro punto importante es la carencia de análisis de manera sistemática y objetiva al plan en curso o culminado, de tal manera que impide determinar el impacto que tendrá frente a la población.

Ante ello, se determina que la deficiencia en los programas presupuestales, ejecución presupuestal, seguimiento y evaluación afecta negativamente a la gestión presupuestal.

A fin de conocer los reportes de los documentos de gestión presupuestal: SIAF, POI, PEI, Información de Personal, Información de casos jurídicos y auditorías, se realizó la siguiente tabla:

\section{Tabla 3}

Reporte de gestión presupuestal

\begin{tabular}{cll}
\hline \multicolumn{1}{c}{ Ítems } & \multicolumn{1}{c}{ Indicador } & \multicolumn{1}{c}{ Valor a registrar } \\
\hline 1 & $\begin{array}{l}\text { Monto asignado a programas presupuestales (Defensa } \\
\text { Jurídica) }\end{array}$ & $2^{\prime} 346^{\prime} 546.00$ \\
2 & Monto en soles de los programas presupuestales & 456 '675.00 PIA \\
\hline
\end{tabular}




\begin{tabular}{|c|c|c|}
\hline 3 & $\begin{array}{l}\text { Número de diseños causales en los programas } \\
\text { presupuestales }\end{array}$ & 4 ( trimestral) \\
\hline 4 & Monto del presupuesto en el POI (PIA y PIM) & $1 ' 540{ }^{\prime} 541.00$ \\
\hline 5 & Calendario del gasto por trimestres & $700 ' 231.00$ \\
\hline 6 & Número de procesos programados en PAC & 12 \\
\hline 7 & Número de procesos atendidos en PAC & 9 \\
\hline 8 & $\begin{array}{l}\text { Número de monitoreo a indicadores de programas } \\
\text { presupuestales }\end{array}$ & 5 (bimestral) \\
\hline 9 & Número de reportes de indicadores por programas & 2 \\
\hline 10 & $\begin{array}{l}\text { Número de directivos involucrados en el seguimiento } \\
\text { presupuestal }\end{array}$ & 3 \\
\hline 11 & $\begin{array}{l}\text { Número de evaluaciones realizadas a los programas } \\
\text { presupuestales }\end{array}$ & 10 \\
\hline 12 & $\begin{array}{l}\text { Número de retroalimentaciones de programas en } \\
\text { función de evaluación }\end{array}$ & 4 \\
\hline 13 & Número de planes operativos & 1 \\
\hline 14 & Número de abogados asignados a la defensa jurídica & 10 \\
\hline 15 & $\begin{array}{l}\text { Número de personal auxiliar asignado a la defensa } \\
\text { jurídica }\end{array}$ & 8 \\
\hline 17 & $\begin{array}{l}\text { Monto de asignación presupuestal para procesos } \\
\text { administrativos de la defensa jurídica }\end{array}$ & $125^{\prime} 549.00$ \\
\hline 18 & Número de casos en cumplimiento de sentencia & 25 \\
\hline 19 & Número de casos con apersonamiento & 8 \\
\hline 20 & Número de reportes de indicadores por programas & 4 (trimestral) \\
\hline 21 & $\begin{array}{l}\text { Número de directivos involucrados en el seguimiento } \\
\text { presupuestal }\end{array}$ & 4 \\
\hline 22 & $\begin{array}{l}\text { Número de evaluaciones realizadas a los programas } \\
\text { presupuestales por funciones y genéricas del gasto }\end{array}$ & 102 \\
\hline 23 & $\begin{array}{l}\text { Número de retroalimentaciones de programas en } \\
\text { función de evaluación }\end{array}$ & 6 \\
\hline
\end{tabular}

Fuente: Análisis de la guía documental. 
La recolección de datos permitió conocer la gestión presupuestal, donde el número de diseños causales en los programas presupuestales son 4 de manera trimestral. El número de procesos programados en PAC es 12 de manera mensual a fin de alcanzar las metas establecidas, con subgrupos. El número de procesos atendidos en PAC es 9 ejecutadas en su totalidad. El número de monitoreo a indicadores de programas presupuestales es 5 de manera bimestral. Del mismo modo, el número de reportes de indicadores por programas es 2; los cuales se efectúan de manera semestral con la finalidad de efectuar un lineamiento de las metas. El número de evaluaciones realizadas a los programas presupuestales por funciones y genéricas del gasto es 10 , de las cuales se han agrupado de acuerdo a los lineamientos y programas establecidos. Para el número de retroalimentaciones de programas en función de evaluación, se ha desarrollado 4 procesos de retroalimentación en lo que va del año, estos orientados al mejoramiento en los procesos de entornos virtuales, y sobre todo en la mitigación de la Covid-19.

El número de casos en cumplimiento de sentencia es 25 , lo cual ha disminuido, por motivo del aislamiento social. Por otro lado, el número de casos con apersonamiento es 8 de acuerdo a la Codiv-19, se disminuyó la presencia al momento de presentar procesos documentarios. Por último, el número de retroalimentaciones de programas en función de evaluación es 6 en lo que va del año, algunos se retrasaron por la COVID-197.

Un análisis general de la información, de acuerdo al diagnóstico que se ha efectuado, resulta prudente el desarrollo de un modelo en la que se tenga establecido criterios para el mejoramiento de la organización.

\section{CONCLUSIÓN O CONSIDERACIONES FINALES}

El estudio presenta mediante un diagnóstico la realidad de como se viene desarrollando dentro de la institución contribuyendo de esta manera en la mejora de la defensa jurídica, por cuanto este representa un proceso elemental, además de conllevar una gran responsabilidad a favor de la institución.

\section{LISTA DE REFERENCIAS}

Acuña, R. y Ángeles, K. (2017). La defensa jurídica del Estado: Optimización del rendimiento de la Procuraduría General (Artículo científico). Revista de la Universidad del Pacífico, Perú. http://repositorio.up.edu.pe/handle/11354/1977 
Aguado, J. y López, A. (2016). Cost and price auditing: effectiveness in the procurement of defense services in Spain. (Artículo científico). Revista de administración $\begin{array}{lllllll}\text { pública. } & 50 & \text { (5), } & \text { p. } & 745 & - & 764 .\end{array}$ https://www.redalyc.org/pdf/2410/241048305003.pdf

Aguilar, N. y Díaz, A. (2020). Restricciones para orientar a resultados los programas de desarrollo rural en México. (Artículo científico). Revista Agricultura, sociedad y $\begin{array}{llllllll}\text { desarrollo. } & 16 & (29), & \text { p. } & 1 & - & 15 . & \text { DOI: }\end{array}$ http://www.scielo.org.mx/scielo.php?script=sci_arttext\&pid=S187054722019000 200199\&lng=es\&nrm=iso

Arana, N. y Ramírez, N. (2019). Participative management model for humanizing public spaces. Analco neighborhood, Puebla, Mexico1. (Artículo científico). Revista Bitácora Urbano Territorial. 29 (1), p. 1 - 15. DOI: https://www.redalyc.org/jatsRepo/748/74858283005/74858283005.pdf

Arieta, J. (2017). Rise and Fall of the Constitutional Right to a Jury Trial for Criminal Cases in the United States. (Artículo científico). Revista de Derecho. 2 (78), p. 1 - 24. DOI:

http://www.scielo.org.pe/scielo.php?script=sci_arttext\&pid=S0251342020170001 00006

Arrais, T. (2019). Income Inequality, Public Employment and Income Transfers In Contemporary Brazil. (Artículo científico). Revista Mercator. 18 (3), p. 1 - 20. DOI: $\quad$ https://www.scielo.br/scielo.php?pid=S198422012019000100206\&script=sci_arttext\&tlng=en

Bernal, N. (2016). Los gastos públicos en pensiones en América Latina y sus proyecciones al año 2075: evidencia de Chile, Perú, Colombia y México. (Artículo científico). Revista Apuntes. 43 (79), p. $80 \quad-\quad$ 128. DOI: http://www.scielo.org.pe/pdf/apuntes/v43n79/a03v43n79.pdf

Cantuarias, P. (2014) La Situación Jurídica de la Defensa del Estado: El Caso Latinoamericano. España: Editorial. Mater Legis

Cano, E. y Chamizo, J. (2018). Estabilidad presupuestaria, financiamiento y responsabilidad social en los municipios españoles. (Artículo científico). Revista Contaduría y Administración. 63 (3), $\quad$ p. $1 \quad-\quad$ 14. DOI: 
http://www.scielo.org.mx/scielo.php?script=sci_arttext\&pid=S018610422018000 400012\&lang=pt

Carrillo, F. y Varela, F (2016) Hacia un Sistema Integrado de Defensa Jurídica Estatal: situación y perspectivas de una política pública en Colombia. (Artículo científico). Revista del CLAD Reforma y Democracia, núm. 56. DOI: https://www.redalyc.org/pdf/3575/357533688005.pdf

Carvalho, I. y Mazzon, J. (2019). Thresholds Are Everywhere: A Systems Approach to Public Policy. (Artículo científico). Revista Brazillian Administration Review. 16 (3),

p. 1

24.

DOI: https://www.redalyc.org/jatsRepo/841/84162090001/84162090001.pdf

Cauvin, T. (2018). The Rise of Public History: An International Perspective. (Artículo científico). Revista Historia Critica. 68 (2), p. 3 - 26. DOI: https://www.redalyc.org/jatsRepo/811/81156122001/81156122001.pdf

CONCYTEC (2018). Reglamento de calificación, clasificación y registro de los investigadores del sistema nacional de ciencia, tecnología e innovación tecnológica reglamento RENACYT. Recuperado de: https://portal.concytec.gob.pe/images/noticias/Propuesta_del_nuevo_Reglamento_ del_investigador.pdf

Cruz, R. (2019). Defensa putativa. Teoría y práctica. Aspectos jurídicos y psicológicos. Doctrina, jurisprudencia, preguntas, casos y problema. (Artículo científico). Revista de fundamentación juridica. 28 (2), p. $465-470 . \quad$ DOI: https://www.redalyc.org/jatsRepo/720/72062650010/72062650010.pdf

De Oliveira, G. (2016). Reflections On Psycho-Legal Practices In The Triage Of The Public Defender's Office Of São Paulo. (Artículo científico). 21 (3), p. 509 - 521. DOI: https://www.redalyc.org/articulo.oa?id=287148579014_2

De Pontes, A. y Corneli, G. (2017). Legal status of animals and proposals for amendments in congress in the Brazilian National Congress. (Artículo científico). Revista $\begin{array}{lllllll}\text { Bioética. } & 25 & (1), & \text { p. } & 191 & - & 197 .\end{array}$ https://www.redalyc.org/articulo.oa?id=361550413021_2

Gallardo, E. (2017). Metodología de la Investigación. (1ª edición). Editorial: Universidad Continental. Ciudad: Huancayo - Perú. 
Gómez, A. (2019). Public Policies Between Theory and Practice. (Artículo científico). Revista Ánfora. $26 \quad$ (46), $\quad$ p. $\quad 1 \quad$ - $14 . \quad$ DOI: https://doi.org/10.30854/anf.v26.n46.2019.561

Grafe, F. (2016) ¿Es efectiva la defensa jurídica del Estado? Perspectivas sobre la gestión del riesgo fiscal en América Latina. (Artículo científico). Publicado por Banco Interamericano de Desarrollo. España. p. 2 - 45. DOI: https://publications.iadb.org/publications/spanish/document/\%C2\%BFEs-efectivala-defensa-jur\%C3\%ADdica-del-Estado-Perspectivas-sobre-la-gesti\%C3\%B3ndel-riesgo-fiscal-en-Am\%C3\%A9rica-Latina.pdf.

Gutiérrez, A. y Peñaloza, V. (2017). Evaluaciones de diseño y ejecución presupuestal, un instrumento del presupuesto por resultados: algunas experiencias aplicadas en salud. (Artículo científico). Revista Peruana de Medicina Experimental y Salud

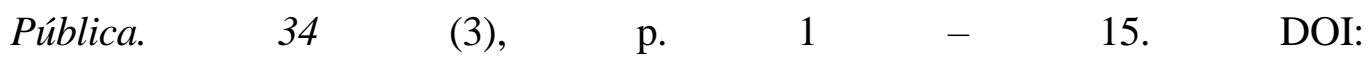
http://www.scielo.org.pe/scielo.php?script=sci_arttext\&pid=S1726463420170003 00020

Gutiérrez, J. (2016) Estructura reglamentaria de la agencia para la defensa jurídica y patrimonial del Estado en Colombia (Artículo científico). Revista Via Luris. 2014 (16), p. 51 - 69. DOI: https://www.redalyc.org/pdf/2739/273933373004.pdf

Guzmán, A. (2016). El Sistema Interamericano de Protección a los Derechos Humanos y la creación de la Agencia Nacional de Defensa Jurídica del Estado en Colombia. (Artículo científico). Revista Justicia Juris. 11 (2), p. 30 - 39. DOI: http://www.scielo.org.co/pdf/jusju/v11n2/v11n2a03.pdf

Herz, M. (2018). The Defense-Development Nexus: Brazilian Nuclear Policy under the Workers' Party Administrations. (Artículo científico). Revista Brasilera de Política $\begin{array}{llllllll}\text { internacional. } & 61 & (1), & \text { p. } & 1 & - & 20 . & \text { DOI: }\end{array}$ https://www.redalyc.org/jatsRepo/358/35860342005/35860342005.pdf

Juárez, M. (2016). El Sistema de Defensa Pública del Estado. Perú: Editorial Planeta Kaneberg, E. y Jensen, L. (2019). Voluntary defense networks in emergency preparedness in developed countries: the case of Sweden. (Artículo científico). Revista Científica General José María Córdova. 17 (26), p. 228 - 250. DOI: https://www.redalyc.org/jatsRepo/4762/476263164002/476263164002.pdf 
Klein, L. y Lemos, R. (2019). Quality of working life: parameters and evaluation in the public service. (Artículo científico). Revista de Administración Mackenzie. 20 (3), p. 1 - $35 . \quad$ DOI: https://www.redalyc.org/jatsRepo/1954/195460883004/195460883004.pdf

Mafaldo, A. (2017). Gestión del Sistema de Defensa Jurídica del Estado y los procesos contenciosos administrativos en la Unidad Ejecutora 301 - Educación Bajo Mayo - San Martín, 2016 (Tesis de maestría). Universidad César Vallejo, Perú. Recuperado de: http://repositorio.ucv.edu.pe/handle/UCV/12751

Martin, V. (2016). Análisis de los procedimientos judiciales suscitados por la deficiente defensa jurídica. Estudio del caso: Proceso penal conforme a lo tipificado en la Ley N906. (Artículo científico). Revista Científica de la Universidad Católica de Colombia, Colombia.

DOI: https://repository.ucatolica.edu.co/bitstream/10983/21508/1/Nulidad\%20de\%20lo s\%20procesos\%20judiciales\%20por\%20deficiente\%20defensa\%20jur\%C3\%ADd ica.pdf

Monje, C. (2015). Metodología de la Investigación Cuantitativa y Cualitativa. ( $1^{\text {a }}$ edición). Editorial: Universidad Surcolombiana. Ciudad: Neiva

Pachot, K. (2016). El derecho constitucional al deporte en la doctrina y el derecho comparado. (Artículo científico). Revista de cuestiones constitucionales. 2 (35), p. 1

22.

DOI: http://www.scielo.org.mx/scielo.php?script=sci_arttext\&pid=S140591932016000 200119

Parra, G. (2017). Evaluación de la política de defensa jurídica del Estado Colombiano, 2002-2014. (Artículo científico). Revista de la Universidad Libre de Colombia, Colombia.

DOI: https://repository.unilibre.edu.co/bitstream/handle/10901/11096/Documento\%20fi nal\%20enero\%2025\%202016\%20revisado.pdf?sequence=1\&isAllowed=y

Piña, T. (2017). Defensa pública del Estado y sus implicancias en la gestión de la Municipalidad de Maynas, año 2016. (Artículo científico). Revista de la Universidad Nacional de la Amazonía Peruana, Perú. DOI: http://repositorio.unapiquitos.edu.pe/bitstream/handle/UNAP/5385/Julio_Tesis_Ti tulo_2017.pdf?sequence=1\&isAllowed=y 
Roitbarg, H. (2018). La rigidez del gasto público brasileño a largo plazo. (Artículo científico). Revista Apuntes del Cenes. 37 (66), p. 1 - 16. DOI: http://www.scielo.org.co/scielo.php?script=sci_arttext\&pid=S0120305320180002 00077

Saavedra, J. (2017). La defensa jurídica del Estado y su relación con la corrupción y la función pública del Gobierno Regional de San Martín, año 2016 (Tesis de maestría). Universidad César Vallejo, Perú.

Sampieri, R. (2014). Metodología de la investigación. (6º ed.). Editorial Mc Graw Hill: México. Recuperado de: https://www.uca.ac.cr/wpcontent/uploads/2017/10/Investigacion.pdf

Stella, L. (2017). Transformaciones del Estado y cambios en la administración pública. Tendencias teóricas de la administración pública. (Artículo científico). Revista A \& D. 47 (2), 36 - 51. DOI: https://dialnet.unirioja.es/descarga/articulo/6403423.pdf

Urrego, G. (2016). Análisis de la defensa jurídica y patrimonial del Estado Colombiano: Un desafío colectivo y gubernamental (Artículo científico). Revista de la Universidad Nacional de Antioquía, Colombia. DOI: file:///www.una.edu.co.//LaDefensaJuridicaYPatrimonialDelEstadoColombiano5151533.pdf

Vásconez, M. (2017). Una defensa del racionalismo jurídico, Madrid, Plaza y Valdés. (Artículo científico). Revista de Fundamentación juridica. 27 (1), p. 139 - 143. DOI: https://www.redalyc.org/jatsRepo/720/72056648008/72056648008.pdf

Valderrama, S. (2016). Pasos para elaborar proyectos de investigación científica. ( $\left.1^{\circ} \mathrm{ed}.\right)$. Perú: Editorial San Marcos.

Villar, M. (2016). Condiciones del ejercicio de defensa jurídica en la fase de instrucción y el trabajo del defensor de oficio. (Tesis de maestría). Universidad Nacional Mayor de San Marcos, Perú. Recuperado de: https://cybertesis.unmsm.edu.pe/bitstream/handle/cybertesis/209/Villar_rm.pdf?se quence $=1$ 